\title{
Development of a framework for critical care resource allocation for the COVID-19 pandemic in Saskatchewan
}

\author{
Sabira Valiani MD, Luke Terrett MD, Colin Gebhardt MD, Oksana Prokopchuk-Gauk BSc MD, \\ Melody Isinger BA MA DHCE
}

Cite as: CMAJ 2020 September 14;192:E1067-73. doi: 10.1503/cmaj.200756

pot n March 2020, health authorities throughout Canada developed frameworks for resource allocation to address potentially overwhelming demand for critical care resources because of coronavirus disease 2019 (COVID-19). We describe the ethical principles and process used in Saskatchewan to develop an operational resource allocation framework for adult patients.

\section{What are the key ethical principles for resource allocation in a pandemic?}

In a pandemic, common ethical principles may be weighed differently than in usual practice. Generally, the decision to institute, continue, withhold or withdraw life-sustaining therapies is based on the interplay of medical prognosis and patient autonomy. In pandemic situations, autonomy considerations are considered secondary to distributive justice. ${ }^{1}$ The principles of respect for human dignity and nonabandonment must also be upheld, in particular for patients who are excluded from care in the intensive care unit (ICU). These patients should be provided appropriate palliative care..$^{2,3}$

During implementation of a resource allocation protocol, the following process principles must be upheld: transparency, consistency, accountability, proportionality and responsiveness..$^{4-6}$ It is necessary to develop an a priori triage protocol that uses objective physiologic criteria upon which resource allocation decisions can be made. Our approach to developing a resource allocation protocol is detailed in Box 1.

The goal of resource allocation protocols is to identify patients whose outcome would be fatal if ICU care is denied, but who are likely to survive if they do receive ICU care..$^{1,7}$ In other words, the protocol seeks to find patients for whom a substantial "incremental" survival benefit would be derived by admission to the ICU. ${ }^{8}$ Although allocation to maximize survival to hospital discharge is considered the most acceptable principle, ethical discourse and public consultation has shown that other allocation principles may be also acceptable. ${ }^{9,10}$ These principles include saving the most life-years (prioritizing those who are

\section{KEY POINTS}

- The coronavirus disease 2019 (COVID-19) pandemic raised the possibility of overwhelming demand for critical care resources, necessitating the creation of resource allocation frameworks.

- The working group who developed Saskatchewan's resource allocation framework were guided by the ethical principles of transparency, consistency, accountability, proportionality and responsiveness.

- Saskatchewan's framework was designed to include considerations that will not unfairly disadvantage older adults, people who are disabled and those with chronic health conditions; a goal of maximizing survival to hospital discharge rather than life-years saved; and a triage team at arms-length from the most responsible physician directly involved in the care of the patient who is critically ill.

- The framework outlined objective and increasingly stringent criteria for exclusion from care in the intensive care unit proportional to the capacity strain on the health system, intended to be used by a representative triage team to make decisions under the oversight of a dedicated committee.

- As the COVID-19 pandemic subsides, priority should be given to seeking broad consultation to elicit public perspectives on resource allocation for health care in crises, which should inform future frameworks.

most likely to live the longest after recovery), life-stage or lifecycle criteria (prioritize patients who have not had the opportunity to live through stages of life such as developing a career or having children) and instrumental value (prioritize those who have particular instrumental value to others in the pandemic, e.g., health care workers).

We designed our framework to maximize survival to hospital discharge, given its acceptance in other resource allocation frameworks and studies. ${ }^{7,9,11}$ Members of our working group agreed that maximizing life-years saved could potentially unfairly disadvantage older adults, patients with disabilities and patients with chronic conditions. However, criteria were included within the framework that could serve as tiebreakers, 


\section{Box 1: Development of the framework}

- A working group of physicians (representing critical care, internal medicine, cardiology, infectious diseases, surgery, trauma and transfusion medicine), nurses, departmental leadership, health care administrators, ethicists and a patient-family advisor was convened to develop the framework.

- One author (S.V.) performed a literature review using purposive sampling of articles in English relevant to the following topics: triage guidelines for mass-casualty events or pandemics; triage protocols in the United States or Canada, from peer-reviewed and grey literature; bioethical discourse on principles of resource allocation; performance of triage protocols in practice; and information about prediction of clinical course and outcome for coronavirus disease 2019 (COVID-19). Reference lists from pertinent papers were reviewed to find additional relevant articles, and relevant references were solicited from the working group.

- The working group met virtually 3 times over about 4 weeks. A draft framework was circulated to members of the working group before the first meeting, based on the literature review, the clinical expertise of intensivists within the working group and knowledge about the health care system in Saskatchewan. Open discussions at the virtual meetings led to group consensus for all aspects of the framework, in particular for the criteria to be used to exclude patients from critical care. Conservative exclusion criteria were used in an effort to err on the side of the rule of rescue. We also used consultants when necessary, including those with expertise in geriatrics, neurology and emergency medicine.

in the following order of priority: physiologic criteria, life-cycle and instrumental value criteria (Figure 1, Table 1). Tiebreaker criteria would be used when 2 patients present with identical clinical and physiologic situations. For example, if a healthy 50-year-old and a healthy 70-year-old both require ICU care and have a Sequential Organ Failure Assessment (SOFA) score of 5, the life-year tiebreaker criterion would be used to offer the 50-year-old an ICU bed first.

Importantly, quality-of-life considerations should not factor into resource allocation decisions. ${ }^{12}$ First, quality-of-life judgments may unfairly disadvantage older adults, patients who require long-term ventilation and patients with disabilities. Second, during a pandemic, it may not be possible to determine future quality of life before the institution of life-supportive therapy owing to severe time and resource constraints. ${ }^{10}$ Most importantly, quality-of-life considerations are most relevant in the shared decision-making paradigm, where the patient may refuse care because it would leave them with an unacceptable quality of life. The acceptability of a certain quality of life is solely the patient's purview. In pandemic situations where autonomy is not at the forefront of ethical decision-making, the patient may refuse ICU care, but physicians should not make this judgment on behalf of the patient unilaterally.

Special populations such as older adults, patients who are undergoing long-term ventilation, health care workers, patients who are pregnant and patients with disabilities require specific considerations and solutions (Table 2).

\section{How was Saskatchewan's Critical Care Resource Allocation Framework operationalized?}

The first draft of our resource allocation framework was largely based on the Ontario Health Plan for an Influenza Pandemic (OHPIP), ${ }^{7}$ which describes inclusion and exclusion criteria for ICU care and a prioritization tool. A similar approach has been adopted in numerous subsequent guidelines and resource allocation frameworks. ${ }^{2,11,21-24}$ Therefore, because of ease of implementation and broad acceptance in the literature, we chose the OHPIP as a starting point.

Four major structures and processes were created to operationalize the Saskatchewan Critical Care Resource Allocation Framework.

\section{Province-wide referral system}

If implemented during a pandemic surge, our resource allocation framework will be applied to all adult patients requiring critical care services within Saskatchewan, not just those presenting with COVID-19, to ensure consistency of care for patients regardless of presenting illness. A centralized provincial flow and consultation service was established and will be used to track availability of critical care beds and attempt to balance admissions among tertiary critical care sites.

\section{Stages of critical care triage}

Most of the published triage protocols assume a state of absolute scarcity in which demand outstrips supply of resources. However, as community spread occurs in a pandemic, there may be times when a relative scarcity of resources will exist. Relative scarcity occurs when baseline capacity for critical care is overwhelmed and surge capacity attempts to meet demand. In these stages of relative scarcity, measures to cope with the surge in demand can be implemented that are proportional to the level of scarcity. ${ }^{25}$ Therefore, we developed objective and increasingly stringent criteria for exclusion from ICU care that were proportional to the capacity strain on the system in Saskatchewan (Table 1, Table 3). The effect of this is 3-fold: it ensures that resource allocation decisions are made transparently and consistently throughout all phases of the pandemic (reduces bedside rationing); it forms part of the surge response in attempting to maximize the number of ICU-days available as demand is expected to grow; and it attempts to remain prepared for sudden surges of a large number of patients, such as those seen in some regions of Italy that experienced a severe COVID-19 outbreak. ${ }^{26}$

Our critical care triage stages are compared in Table 3, and detailed exclusion criteria are included in Table 1 . We defined these stages as percentages of baseline capacity of funded ICU beds, because surge capacity is a moving target. The definition of each stage was matched to what surge resources would be required and to the degree to which the system would be strained for manpower, equipment and physical space. For example, 1 of Saskatchewan's tertiary ICUs is funded for 10 beds but can physically accommodate 15 patients. At $150 \%$ of baseline capacity (15 beds, which would correspond to stage 2), a second unit would need to be opened, resulting in a substantial incremental demand for ICU resources. 


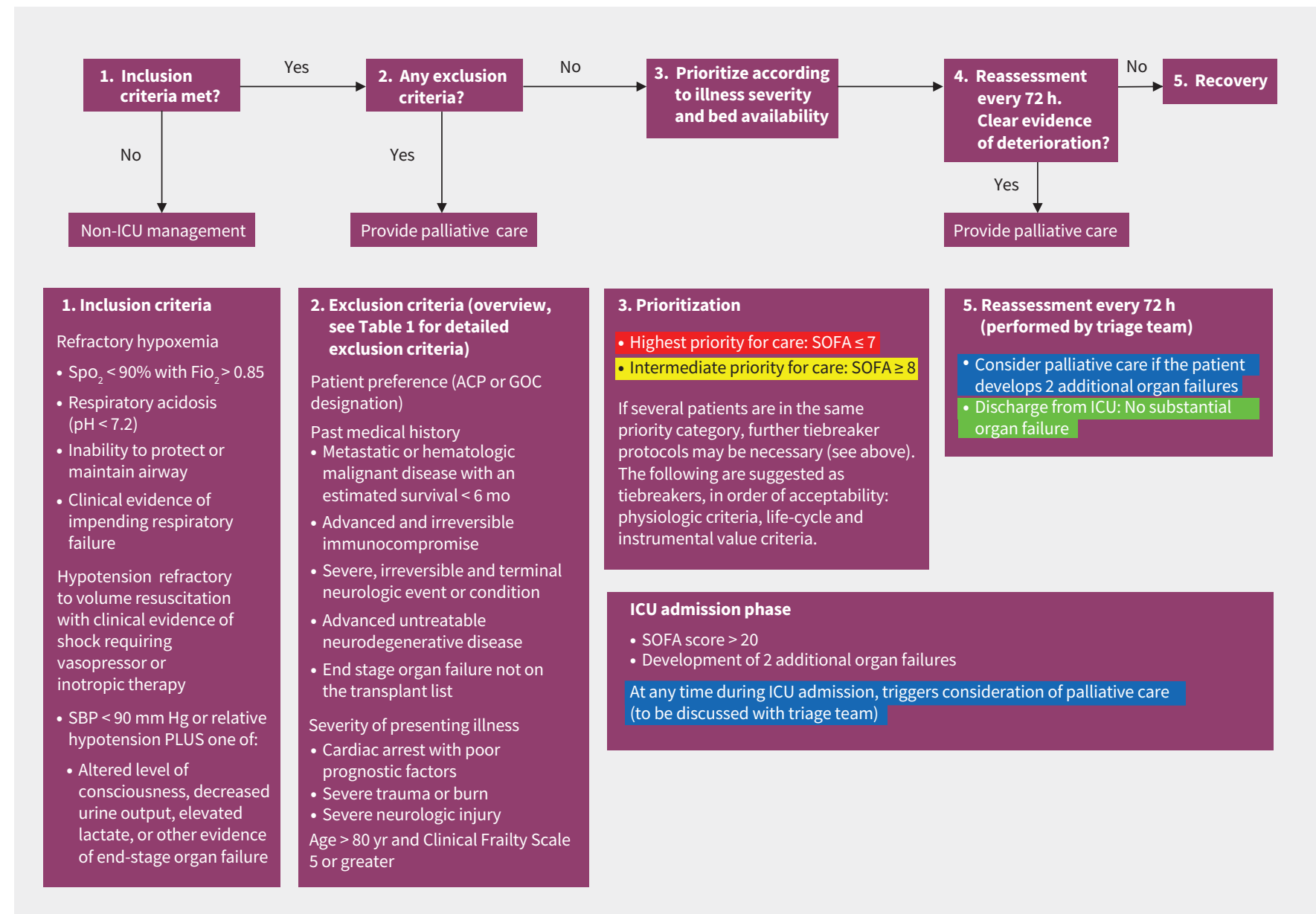

Figure 1: Critical care triage stage 4 protocol for patients with coronavirus disease 2019 in Saskatchewan. Note: $\mathrm{ACP}=$ advance care planning, Fio ${ }_{2}=$ fraction of inspired oxygen, $\mathrm{GOC}=$ goals of care, SOFA = Sequential Organ Failure Assessment, $\mathrm{SpO}_{2}=$ oxygen saturation. Extracorporeal life support (ECLS) may provide effective treatment for refractory cases, but it requires extensive resources. Each request for ECLS will be reviewed by at least 2 ECLS experts, in addition to the triage team. These ECLS experts will be designated by the area leads of the Department of Critical Care (Regina and Saskatoon). The number of patients that can be placed on ECLS is small and should be decided on a case-by-case basis. Definite exclusion criteria include age older than 60 years; receiving mechanical ventilation for more than 7 days; irreversible neurologic, multiorgan failure; malignancy; cardiac arrest; severe end-stage liver, lung, kidney or heart disease; advanced neurocognitive disease; pregnancy; body mass index > 45; inability to receive anticoagulation or blood products; or ECLS resources not available in city. Legend: red = highest priority patient (most likely to benefit from admission to the intensive care unit [ICU]), yellow = intermediate priority patient (may benefit from ICU care), green = patient does not require ICU care (too well), blue = palliative care only (poor prognosis is likely).

\section{Triage team structure}

In a scenario requiring activation of our framework, every patient who may need ICU care will be reviewed by a triage team consisting of 2 intensivists and an ethicist, ideally representing at least 2 geographic locations. The working group felt that this structure for the triage team, along with standardized documentation, would ensure consistency and accountability. The triage team is convened by teleconference via centralized services within the province-wide referral system to achieve consistency of the resource allocation process for patients in both rural and urban areas. The use of teleconference has an added benefit of reducing hidden bias for vulnerable populations, as teleconferenced triage team members are provided only with anonymized clinical data. Further, this structure ensures that the most responsible physician, who has a fiduciary duty to their patient, is not involved in rationing decisions. The most responsible physician is therefore not placed in a conflict-of-interest situation and can continue to advocate for the patient. For example, if the most responsible physician believes a factual error has been made, or if the patient's status changes at a later date, the most responsible physician can ask for a review of the triage team's decision.

\section{Oversight committee}

An oversight committee was established with the goal of ensuring consistency, accountability and responsiveness. The committee includes representation from physicians, patients and families, Saskatchewan Health Authority First Nations and Metis Health Services, rural health care workers, a lawyer and clinical ethicists. This committee is responsible for updating the resource allocation protocol as new local clinical data or published evidence become available. A formal evidence support team was created to support the evidence-based revision of 
Table 1: Detailed exclusion criteria

\section{Criteria} Critical care triage stage 1

Patient preference

As documented by goals-of-care and advance care planning discussions

Past medical Clinician judgment; must history be mutually agreed upon by patient and clinician
Severity of presenting illness
Clinician judgment; must be mutually agreed upon by patient and clinician
Age and

Clinical

Frailty Score

\section{Critical care triage stage 2}

Critical care triage stages 3 and 4

As documented by goals-of-care and advance care planning discussions

- End-stage organ failure*

- Heart failure NYHA class IV

- Lung disease

- COPD with FEV1 $<30 \%$ predicted or baseline $\mathrm{PaO}_{2}<55 \mathrm{~mm} \mathrm{Hg}$, or secondary pulmonary hypertension

- Cystic fibrosis with postbronchodilator FEV $1<30 \%$ predicted or baseline $\mathrm{PaO}_{2}<55 \mathrm{~mm} \mathrm{Hg}$

- Pulmonary fibrosis with VC or TLC $<60 \%$ predicted, baseline $\mathrm{PaO}_{2}<55 \mathrm{~mm} \mathrm{Hg}$ or secondary pulmonary hypertension

- Primary pulmonary hypertension with NYHA class IV symptoms

- Cirrhosis with MELD $>20$
- Age $>80$ yr and cardiac arrest with 1 of the following poor prognostic factors: $\dagger$

- Unwitnessed cardiac arrest

- Any PEA arrest

- Recurrent cardiac arrest
As documented by goals-of-care and advance care planning discussions

- Metastatic malignant disease with expected survival of $<6$ mo

- Advanced and irreversible immunocompromise

- Severe, irreversible and terminal neurologic event or condition (end-stage dementia)

- Advanced untreatable neurodegenerative disease (Parkinson disease, ALS)

- End-stage organ failure*

- Heart failure NYHA class III or IV

- Lung disease

- COPD with FEV1 $<30 \%$ predicted or baseline $\mathrm{PaO}_{2}<55 \mathrm{~mm} \mathrm{Hg}$, or secondary pulmonary hypertension

- Cystic fibrosis with postbronchodilator FEV1<30\% predicted or baseline $\mathrm{PaO}_{2}<55 \mathrm{~mm} \mathrm{Hg}$

- Pulmonary fibrosis with VC or TLC $<60 \%$ predicted, baseline $\mathrm{PaO}_{2}<55 \mathrm{~mm} \mathrm{Hg}$ or secondary pulmonary hypertension

- Primary pulmonary hypertension with NYHA class IV symptoms

- Cirrhosis with MELD > 20

- Cardiac arrest, regardless of age, with 1 of the following poor prognostic factors:

- Unwitnessed cardiac arrest

- Any PEA arrest

- Recurrent cardiac arrest

- Severe trauma or burns

- Trauma with ISS > 16, unless determined to be acutely reversible

- Burns with 2 of the following:

- Age $>60 \mathrm{yr},>40 \%$ BSA, inhalational injury

- Severe neurologic injury

- TBI meeting all of the following criteria:

- Age $>60 \mathrm{yr}, \mathrm{GCS}<8$, and 1 or both unreactive pupils

- SAH with WFNS grade $V$

- CVA

- Age > 70 yr with large MCA territory CVA, substantial deficits, not amenable to reperfusion

- Posterior circulation stroke with GCS $<8$

- Age $>80$ yr and

- Clinical Frailty Score $\geq 5$

Note: $\mathrm{ALS}=$ amyotrophic lateral sclerosis, BSA = body surface area, $\mathrm{COPD}=$ chronic obstructive pulmonary disease, $\mathrm{CVA}=$ cerebrovascular accident, $\mathrm{FEV} 1=$ forced expiratory volume in 1 minute, GCS = Glasgow Coma Scale, ICU = intensive care unit, ISS = Injury Severity Score, MCA = middle cerebral artery, MELD = Model for End-Stage Liver Disease, NYHA = New York Heart Association, $\mathrm{PEA}=$ pulseless electrical activity, $\mathrm{SAH}=$ subarachnoid hemorrhage, $\mathrm{TBI}=$ traumatic brain injury, $\mathrm{TLC}=$ total lung capacity, VC = vital capacity, WFNS = World Federation of Neurosurgical Societies.

*If the patient is currently on a waiting list for organ transplant and admission to the ICU would place them at the top of the waiting list, an exception should be made and the patient should be admitted to the ICU. However, if organ donation programs are put on hold because of the pandemic, this exception is no longer valid.

†If the patient's most responsible physician determines the cause of the cardiac arrest to be acutely reversible, the patient is not excluded from ICU care.

this protocol, and any clinician could provide this team with evidence that could inform a revision. Further responsibilities of the committee include mediating disagreements among members of the triage team, reviewing appeals by the most responsible physician and performing retrospective qualityimprovement reviews.

\section{What are the limitations of our approach?}

Because of the rapid trajectory of the COVID-19 pandemic in early 2020, the Saskatchewan Critical Care Resource Allocation Framework was developed expediently. There are shortcomings with this process that we are working to improve. 


\section{Population}

Older adults

Chronically ventilated

Patients with disabilities

Pregnant patients

Health care workers
Rationale

Incorporation into the Saskatchewan Critical Care Resource Allocation Framework

A combination of age and frailty is used as exclusion criteria in stage 3 (Table 1 ). exclusively by age. ${ }^{13}$ Instead, frailty (defined as CFS $\geq 5$ ) is associated with higher in-hospital and long-term mortality. ${ }^{14}$ In octogenarians, frailty is predictive of short-term ICU mortality. ${ }^{15,16}$ Although frailty may portend a poorer outcome in younger patients who are critically ill, the validity of applying the CFS more broadly is still being investigated. ${ }^{17}$

Palliation for patients on long-term ventilation is not justifiable, even if it allows a greater number of healthier patients to survive. This policy would risk inappropriate quality-of-life judgments and could be seen as disadvantaging vulnerable patients.

Patients with intellectual, physical or developmental disabilities are considered vulnerable populations and at risk for discrimination within the health care system. ${ }^{18}$ Patients with stable, nonprogressive conditions will not be excluded solely on the basis of these conditions.

A patient who is pregnant and her potentially viable fetus should be considered as 2 separate lives, and therefore these patients can be prioritized based on the life-cycle principle. ${ }^{19}$

Health care workers have instrumental value (i.e., a health care worker who is healthy can save the lives of more patients). ${ }^{10,20}$ However, during a stage of critical illness, it is unclear whether the health care worker, if saved, would be able to return to work in a timely fashion to help others. Instrumental value is a potentially subjective concept that lends itself too easily to other, potentially extraneous, considerations of social worth.

Patients who are already receiving life-sustaining treatments in long-term care settings or at home are considered to be a different population altogether from the medical patient who is acutely ill. However, should patients receiving long-term ventilation require treatment in an acute care facility, they should be considered part of the acute care cohort and subject to the resource allocation framework. ${ }^{2}$

The structure and process of the triage team is meant to form a system of checks and balances to eliminate discrimination based on disability.

Pregnancy with a potentially viable fetus is included as a tiebreaker in stage 4 (Figure 1).

Health care workers are included as tiebreakers in stage 4 (Figure 1).

Table 3: Comparison of critical care triage stages

\begin{tabular}{|c|c|c|c|c|c|}
\hline $\begin{array}{l}\text { Triage } \\
\text { stage }\end{array}$ & $\begin{array}{l}\text { Baseline ICU } \\
\text { capacity, \% }\end{array}$ & Scarcity & Inclusion criteria & Exclusion criteria overview (see Table 1) & $\begin{array}{l}\text { Withdrawal of life- } \\
\text { supportive treatment }\end{array}$ \\
\hline 1 & $100-150$ & Relative & $\begin{array}{l}\text { According to usual } \\
\text { clinical practice }\end{array}$ & According to usual clinical practice & $\begin{array}{l}\text { According to usual clinical } \\
\text { practice }\end{array}$ \\
\hline 2 & $150-200$ & Relative & $\begin{array}{l}\text { According to usual } \\
\text { clinical practice }\end{array}$ & $\begin{array}{l}\text { - End-stage organ failure } \\
\text { - Cardiac arrest with poor prognostic factors }\end{array}$ & $\begin{array}{l}\text { According to usual clinical } \\
\text { practice }\end{array}$ \\
\hline 3 & $200-250$ & Relative & $\begin{array}{l}\text { According to usual } \\
\text { clinical practice }\end{array}$ & $\begin{array}{l}\text { - End-stage organ failure } \\
\text { - Terminal neurologic diseases } \\
\text { - Metastatic malignant disease with poor } \\
\text { prognosis } \\
\text { - Cardiac arrest with poor prognostic factors } \\
\text { - Severe trauma or burns } \\
\text { - Severe neurologic injury } \\
\text { - Age }>80 \text { yr and Clinical Frailty Score } \geq 5\end{array}$ & $\begin{array}{l}\text { According to usual clinical } \\
\text { practice }\end{array}$ \\
\hline 4 & $>250$ & Absolute & $\begin{array}{l}\text { Formal inclusion } \\
\text { criteria (Figure 1) }\end{array}$ & According to stage 3 exclusion criteria & $\begin{array}{l}\text { Formalized reassessments and } \\
\text { uniform criteria for palliation }\end{array}$ \\
\hline
\end{tabular}




\section{Membership in the working group}

The working group (see Box 1) was heavily weighted toward clinicians (physicians and nurses) and health care administrators, which, at a time of uncertainty and potential rapid change in the pandemic, expedited the development of this framework. However, other important stakeholders could have been included in earlier reviews of the framework. Representation from rural and Indigenous stakeholders, and the health authority's legal team, were subsequently included on the oversight committee. The working group sought approval from the Saskatchewan Ministry of Health only after the document had been approved. The Saskatchewan Human Rights Commission requested to review this approved document and provided minimal revisions.

\section{System integration}

Our framework does not specifically address the provision of other life-supportive therapies that might become scarce during a pandemic surge such as dialysis, medications and blood transfusions. Maintaining open communication with parallel committees involved in managing resource-specific shortages, such as blood supply, ${ }^{21}$ is essential.

\section{Public consultation}

Ideally, the goals of a resource allocation framework and the definition of benefit are developed with public consultation. Different groups within Saskatchewan may consider benefit differently. There is a noticeable gap in the literature regarding public input on how to allocate resources in a pandemic, with only 1 study identified by our working group. ${ }^{9}$ As the COVID-19 pandemic subsides, priority should be given to seeking broad public consultation to elicit perspectives on resource allocation for health care, which should inform future frameworks.

\section{How does our COVID-19 resource allocation framework compare with others?}

The first Canadian resource allocation framework made publicly available during the COVID-19 pandemic was the Ontario Health Clinical Triage Protocol for Major Surge in COVID Pandemic (the "Ontario protocol"). ${ }^{27}$ The Ontario protocol differs from our framework in 2 important ways. First, the Ontario protocol implements triaging of patients only when demand has exceeded surge capacity. As Saskatchewan is a smaller province with fewer tertiary care ICUs, increasing capacity, in particular scaling human resources, is more challenging. Our working group considered that linking triage with surge capacity would ensure that beds would be available for healthier patients who may present to critical care later in an outbreak than patients who may present earlier because of chronic underlying conditions. Second, the criteria for palliation differ. In the Ontario protocol, if a triage level (a similar concept to a triage stage in Saskatchewan's framework) is activated, any patients currently in ICU care who meet exclusion criteria for this triage level are thereafter provided palliative care only. In contrast, the Saskatchewan protocol includes definite criteria for palliation in stage 4 only; otherwise, withdrawal of life-sustaining therapy is based on usual clinical practice. The criterion for palliation in the Saskatchewan protocol has been modified to reflect a longer ICU stay for patients with COVID-19 and SOFA scores that reflect a greater than $90 \%$ mortality based on data from the H1N1 epidemic. ${ }^{28,29}$ The Saskatchewan working group considered that the criteria for palliation should be individualized to the patient and kept independent of the capacity strain as much as possible.

After the initial development of our framework, a multiprinciple allocation framework was described and implemented in some American states. ${ }^{13,20}$ Their approach uses a point system to balance multiple resource allocation principles. Notably, this framework does not exclude any patients from ICU care, and patients are prioritized according to those who would benefit most. In contrast, our framework has maintained the primary principle of maximizing patient survival to hospital discharge. Other principles of resource allocation, such as life cycle and instrumental value are used only as tiebreakers, because our working group was concerned that using these principles upfront may inadvertently disadvantage vulnerable patients. A second potential disadvantage of the multiprinciple allocation framework is the lack of exclusion criteria, which may lead to a large number of patients awaiting allocation to life-sustaining treatment. In turn, clinicians may be reticent to provide palliative care until the patient is in extremis at the end of life, for fear that providing palliative treatment for refractory symptoms may shorten life while waiting for an ICU bed. In contrast, frameworks that clearly exclude patients from ICU care may ensure that best medical management is first provided, followed by appropriate palliative care if the patient's condition worsens.

\section{Conclusion}

The Saskatchewan Critical Care Resource Allocation Framework was developed with specific attention to the ethical principles of proportionality, consistency, accountability, transparency and responsiveness. Our context of a small geographically dispersed population required the development of a provincial flow and consultation service to balance capacity strains, as well as development of a triage team structure that is convened through a provincial consultation service, to ensure consistency for rural and potentially vulnerable communities. Describing triage stages, with increasingly stringent exclusion criteria, is an important feature of this resource allocation framework. This staged approach was developed to accompany a surge response in the setting of limited human resources and to reduce bedside rationing.

Ensuring that the process was transparent, consistent, responsive, accountable and proportional were key concerns in the development of this framework. We hope that these key principles, if implemented correctly, will help to alleviate some of the emotional weight of triage decisions on our clinicians and encourage primary health care providers to start discussions about realistic goals of therapy with patients and their families in an outpatient setting to decrease stressful circumstances that they may face if admission to hospital occurs during a pandemic. 


\section{References}

1. Truog RD, Mitchell C, Daley GQ. The toughest triage - allocating ventilators in a pandemic. N Engl J Med 2020;382:1973-5.

2. The Pandemic Influenza Ethics Initiative Work Group of the Veterans Health Administration's National Center for Ethics in Health Care. Meeting the Challenge of Pandemic Influenza: Ethical Guidance for Leaders and Health Care Professionals in the Veterans Health Administration. Washington (DC): National Center for Ethics in Health Care, U.S. Department of Veterans Affairs; 2010:1-83.

3. Arya A, Buchman S, Gagnon B, et al. Pandemic palliative care: beyond ventilators and saving lives. CMAJ 2020;192:E400-4.

4. Biddison LD, Berkowitz KA, Courtney B, et al. Ethical considerations: care of the critically ill and injured during pandemics and disasters: CHEST consensus statement. Chest 2014;146(Suppl):e145S-55S.

5. Hick JL, Pavia AT, Hanfling D, et al. Duty to plan: health care, crisis standards of care, and novel coronavirus SARS-CoV-2 [discussion paper]. Washington (DC): National Academy of Sciences; 2020:1-13.

6. Farmer JC, Wax RS, Baldisseri MR. Preparing your ICU for disaster response. Mount Prospect (IL): Society of Critical Care Medicine; 2012.

7. Christian MD, Hawryluck L, Wax RS, et al. Development of a triage protocol for critical care during an influenza pandemic. CMAJ 2006;175:1377-81.

8. Gomersall CD, Joynt GM. What is the benefit in triage? Crit Care Med 2011; 39:911-2.

9. Biddison ELD, Gwon HS, Schoch-Spana M, et al. Scarce resource allocation during disasters: a mixed-method community engagement study. Chest 2018;153:187-95.

10. Emanuel EJ, Persad G, Upshur R, et al. Fair allocation of scarce medical resources in the time of COVID-19. N Engl J Med 2020;382:2049-55.

11. Christian MD, Devereaux AV, Dichter JR, et al.; Task Force for Mass Critical Care. Introduction and executive summary: care of the critically ill and injured during pandemics and disasters: CHEST consensus statement. Chest 2014; 146(Suppl):8S-34S. [AU: The title and page range were checked and changed. Please confirm.]

12. Schmidt $\mathrm{H}$. The way we ration ventilators is biased: not every patient has a fair chance. The New York Times 2020 Apr. 15.

13. White $\mathrm{DB}$, Katz $\mathrm{MH}$, Luce JM, et al. Who should receive life support during a public health emergency? Using ethical principles to improve allocation decisions. Ann Intern Med 2009;150:132-8.

14. Muscedere J, Waters B, Varambally A, et al. The impact of frailty on intensive care unit outcomes: a systematic review and meta-analysis. Intensive Care Med 2017;43:1105-22.
15. Flaatten $\mathrm{H}$, Lange DW, Morandi A, et al. The impact of frailty on ICU and 30-day mortality and the level of care in very elderly patients ( $\geq 80$ years). Intensive Care Med 2017;43:1820-8.

16. Muessig JM, Nia AM, Masyuk M, et al. Clinical Frailty Scale (CFS) reliably stratifies octogenarians in German ICUs: a multicentre prospective cohort study. BMC Geriatrics 2018;18:162.

17. Bagshaw M, Majumdar SR, Rolfson DB, et al. A prospective multicenter cohort study of frailty in younger critically ill patients. Critical Care 2016;20:175.

18. Savin K, Guidry-Grimes L. Confronting disability discrimination during the pandemic [essay]. Garrison [NY]: The Hastings Center; 2020 Apr. 2:127-33.

19. Daugherty Biddison EL, Faden R, Gwon HS, et al. Too many patients... A framework to guide statewide allocation of scarce mechanical ventilation during disasters. Chest 2019;155:848-54.

20. White $D$, Lo B. A framework for rationing ventilators and critical care beds during the COVID-19 pandemic. JAMA 2020 Mar. 27 [Epub ahead of print]. doi: 10.1001/ jama.2020.5046.

21. Emergency framework for rationing of blood for massively bleeding patients during a red phase blood shortage. National Advisory Committee on Blood and Blood Products; 2012:1-93. Available: www.nacblood.ca/resources/shortages -plan/emergency-framework-final.pdf (accessed 2020 Mar. 26).

22. Joynt GM, Goplan PD, Argent AA, et al. The Critical Care Society of Southern Africa Consensus Guideline on ICU Triage and Rationing (ConICTri). S Afr Med J 2019;109:630-42.

23. Province of British Columbia emergency triage in a pandemic: ventilator allocation framework. British Columbia Critical Care Services; 2012:1-30.

24. Patient care strategies for scarce resource situations. St. Paul (MN): Minnesota Department of Health; 2020:1-50.

25. Christian MD, Sprung CL, King MA, et al.; Task Force for Mass Critical Care. Triage: care of the critically ill and injured during pandemics and disasters: CHEST consensus statement. Chest 2014;146(Suppl):e61S-74S.

26. Grasselli G, Pesenti A, Cecconi M. Critical care utilization for the COVID-19 outbreak in Lombardy, Italy: early experience and forecast during an emergency response. JAMA 2020 Mar. 13 [Epub ahead of print]. doi: 10.1001/jama.2020.4031.

27. Ontario Health. Clinical Triage Protocol for Major Surge in COVID Pandemic March 28 2020. Ontario Health; 2020:1-19.

28. Zhou F, Yu T, Du R, et al. Clinical course and risk factors for mortality of adult inpatients with COVID-19 in Wuhan, China: a retrospective cohort study. Lancet 2020;395:1054-62.

29. Shahpori R, Stelfox HT, Doig CJ, et al. Sequential organ failure assessment in H1N1 pandemic planning. Crit Care Med 2011;39:827-32.
Competing interests: Oksana Prokopchuk-Gauk has received consultant fees from Celgene and Takeda, an honorarium from Canadian Blood Services, and remuneration for conference travel from Octapharma; she is the chair of the National Advisory Committee on Blood and Blood Products, and co-chair of the National Emergency Blood Management Committee. No other competing interests were declared.

This article has been peer reviewed.

Affiliations: Department of Adult Critical Care Medicine (Valiani, Terrett, Gebhardt); Divisions of Transfusion Medicine (Prokopchuk-Gauk) and Ethics (Isinger), Saskatchewan Health Authority; College of Medicine (Valiani, Terrett, Gebhardt, Prokopchuk-Gauk), University of Saskatchewan, Saskatoon, Sask.
Contributors: Sabira Valiani, Luke Terrett, Colin Gebhardt and Oksana Prokopchuk-Gauk acquired the data. All of the authors conceived the work, analyzed and interpreted the data, drafted and revised the manuscript for important intelletual content, gave final approval of the version to be published ad agreed to be accountable for all aspects of the work.

Acknowledgements: The authors thank the members of the working group for their clinical and human expertise to develop the Saskatchewan Critical Care Resource Allocation Framework and this manuscript: Jeffrey Betcher, Jennifer Baird, Eileen Reimche, Sheila Rutledge Harding, Heather Miazga, Vicki Erlich, Kelly Johnson, Paul Hayes, Jan Besse, Erik PausJenssen, Brian Brownbridge and Alastair Wall.

Correspondence to: Sabira Valiani, svaliani@usask.ca 